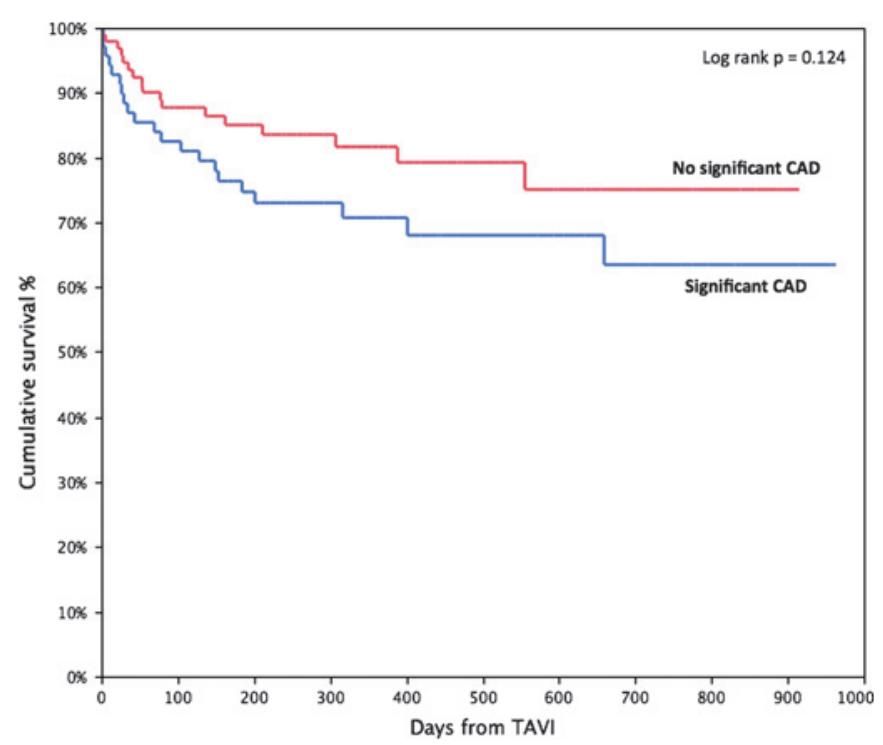

Abstract 26 Figure 1

study. As yet, the impact of PCI to significant CAD upon outcome after TAVI is not known and will be assessed in a prospective, randomised controlled trial currently underway.

\section{PLATELET MONOCYTE AGGREGATES ARE DETERMINANTS OF MICROVASCULAR DYSFUNCTION DURING PERCUTANEOUS CORONARY INTERVENTION FOR STABLE ANGINA AND NON-ST SEGMENT ELEVATION MYOCARDIAL INFARCTION}

doi:10.1136/heartjnl-2011-300198.27

${ }^{1} \mathrm{C}$ A Mavroudis, ${ }^{1} \mathrm{~B}$ Majumder, ${ }^{2} \mathrm{M}$ Lowdell, ${ }^{1} \mathrm{R}$ D Rakhit. ${ }^{1}$ Cardiology Department, Royal Free Hospital, London, UK; ${ }^{2}$ Haematology Department, Royal Free Hospital, London, UK

Background Microvascular dysfunction is associated with adverse outcome in patients with acute coronary syndrome (ACS). During ACS platelet and monocyte derived chemokines, in conjunction with adhesion molecule expression, promote the inflammatory process. Activated platelets express p-selectin which binds to the pselectin glycoprotein ligand on the monocyte forming platelet monocyte aggregates (PMA). PMA expression is a sensitive marker of platelet activation and inflammation. Although platelet monocyte interaction is a normal physiological process, in the presence of platelet activation, activated (CD62+ PMA) may be directly involved in the pathophysiology of intracoronary inflammation and microvascular dysfunction in ACS.

Aim To investigate the relationship between microvascular dysfunction and PMA expression in patients with stable angina and non-ST elevation myocardial infarction (NSTEMI).

Methods Six patients with stable angina undergoing elective PCI and six patients with NSTEMI undergoing non-elective PCI were recruited. Microvascular dysfunction was assessed by measuring the coronary wedge pressure (CwP) and the index of Microvascular resistance (IMR) using a single pressure-temperature sensor-tipped coronary wire from the simultaneous measurement of distal coronary pressure and thermodilution derived mean transit time ( $\mathrm{Tmn}$ ) of a bolus of saline injected at room temperature into the coronary artery during maximum hyperaemia. Blood samples were taken from the coronary artery (distal to the culprit lesion), aorta and the right atrium for PMA estimation. PMAs were assessed using fluorescent monoclonal antibodies and flow cytometry. Total PMAs were calculated and expressed as a percentage of the total monocyte count. Activated CD62+ PMAs were expressed as a percentage of total PMAs.
Results As expected CwP was higher in patients with NSTEMI (46.5 (SD) 18.8) compared with the stable angina patients (Mean (SD) 21.1 (9.3) $\mathrm{p}=0.01$ ). IMR was also higher in patients with NSTEMI (Mean (SD) 27.6 (12.6)) compared with patients with stable angina (Mean (SD) 20.7 (5.4) p=0.2). Total PMAs were nonsignificantly higher in patients with NSTEMI (Mean (SD) 14 (4.8)) compared with stable angina (Mean (SD) 10.9 (4.3) $\mathrm{p}=0.07$ ). CD62 + PMAs were significantly higher in patients with NSTEMI (Mean (SD) 26.9 (12.2)) compared with stable angina (Mean (SD) 13.7 (5.1) $\mathrm{p}=0.02$ ) Abstract 27 figure 1. CwP correlated positively with total PMA $(p=0.01)$ in NSTEMI but not in stable angina patients. However, IMR correlated positively with total PMAs in both stable angina $(p=0.02)$ and NSTEMI $(p=0.08)$ Abstract 27 figure 2.

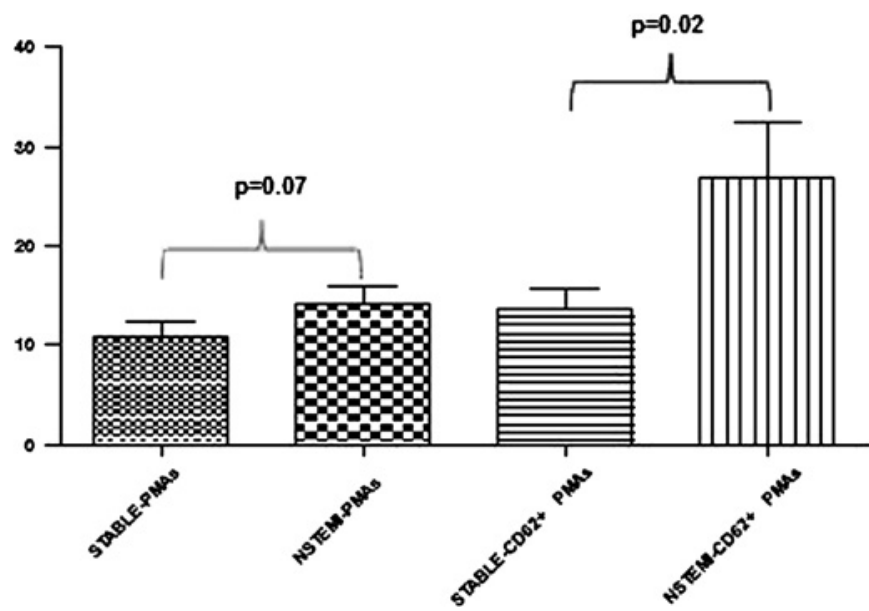

Abstract 27 Figure 1
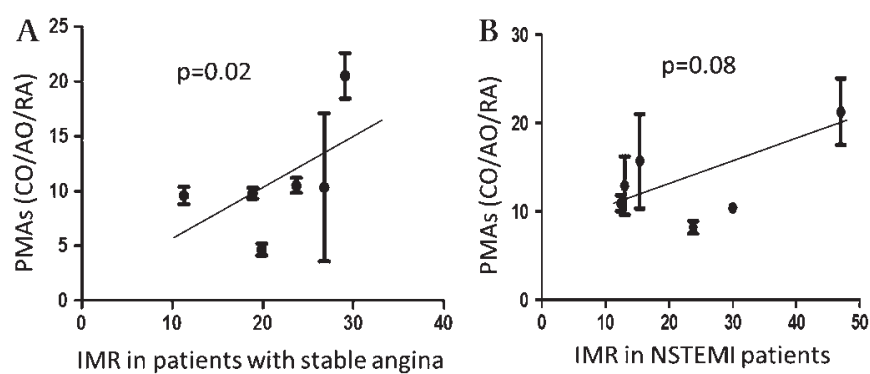

Abstract 27 Figure 2

Conclusions PMAs are elevated in stable coronary disease and ACS with elevated activated CD62+ PMA a hallmark of ACS. PMAs correlate with measured microvascular dysfunction during PCI in stable angina and NSTEMI. This study supports the hypothesis that PMA formation may be important determinants of platelet activation, inflammation and microvascular dysfunction in coronary disease.

\section{LOW FRAME RATE SCREENING DURING PERCUTANEOUS CORONARY ANGIOPLASTY SIGNIFICANTLY REDUCES RADIATION EXPOSURE, GIVES GOOD IMAGE QUALITY WITHOUT AFFECTING PATIENT OUTCOME}

doi:10.1136/heartjnl-2011-300198.28

${ }^{1} \mathrm{~S} J$ Wilson, ${ }^{1} \mathrm{P}$ Venables, ${ }^{2} \mathrm{O}$ Gosling, ${ }^{1} \mathrm{~V}$ Suresh. ${ }^{1}$ South West Cardiothoracic Centre, Plymouth, UK; ${ }^{2}$ Royal Devon and Exeter Hospital, Exeter, UK

Introduction Minimisation of radiation exposure during cardiac procedures is required by statute (IRMER 2000). During coronary angioplasty $47 \%$ of radiation dose is related to screening at standard 